University of Nebraska - Lincoln DigitalCommons@University of Nebraska - Lincoln

$11-2010$

\title{
Time Diary versus Instantaneous Sampling: A Comparison of Two Behavioral Research Methods
}

\author{
Michael Paolisso \\ University of Maryland - College Park, mpaoliss@umd.edu \\ Raymond B. Hames \\ University of Nebraska-Lincoln, rhames2@unl.edu
}

Follow this and additional works at: http://digitalcommons.unl.edu/anthropologyfacpub

Part of the Other Anthropology Commons, and the Social Statistics Commons

Paolisso, Michael and Hames, Raymond B., "Time Diary versus Instantaneous Sampling: A Comparison of Two Behavioral Research Methods" (2010). Anthropology Faculty Publications. 87.

http://digitalcommons.unl.edu/anthropologyfacpub/87

This Article is brought to you for free and open access by the Anthropology, Department of at DigitalCommons@University of Nebraska - Lincoln. It has been accepted for inclusion in Anthropology Faculty Publications by an authorized administrator of DigitalCommons@University of Nebraska Lincoln. 


\title{
Time Diary versus
}

\section{Instantaneous Sampling: A Comparison of Two Behavioral Research Methods}

\author{
Michael Paolisso ${ }^{1}$ and Raymond Hames ${ }^{2}$
}

1 Department of Anthropology, University of Maryland, College Park, MD

2 Department of Anthropology, University of Nebraska-Lincoln, Lincoln, NE

Corresponding author - Michael Paolisso, Department of Anthropology, 1111 Woods Hall, University of Maryland, College Park, MD 20742, USA; email mpaolisso@anth.umd.edu

\begin{abstract}
The accurate collection of unbiased behavioral data is an important component of theory building and ethnographic research. In this article, the authors review two approaches for the collection of behavioral data: time diary and instantaneous sampling. Time diary requires individuals to recall their behavior at specific time intervals; instantaneous sampling relies on researchers observing and recording the behavior of individuals. Each approach has specific strengths and weaknesses. The authors review recent methodological literature on both approaches, identify particular problems with both approaches, and contrast their respective methodological strengths and weaknesses.
\end{abstract}

Keywords: behavioral research, time allocation, time diary method, instantaneous sampling 


\section{Introduction}

The systematic study of human behavior produces invaluable societal and cultural insights. In our field of anthropology, researchers use systematic behavioral research to accomplish a number of research objectives. We study behavior to refine our ethnographic observations (Johnson and Sackett 1998), contribute to theory development (Gurven and Kaplan 2006), generate cross-cultural comparisons (Minge-Klevana 1980; Hames 1989), and use anthropology in applied work (Paolisso et al. 1989; Paolisso et al. 2002).

Despite the centrality of human behavioral research to anthropological (and other social science) research, discussions of the strengths and limitations of different behavioral research approaches has been intermittent. In the 1980s, a small corpus of assessments of anthropology and behavioral research was available to anthropologists interested in different approaches to behavioral research, particularly time allocation research (Gross 1984). Early articles by Johnson $(1975)$ and Baksh $(1989,1990)$ introduced the basics of instantaneous sampling (spot checks), and Johnson and Sackett (1998) later provided an overview of direct systematic observation methods.

More recently, others have discussed observation in the context of data recording software (Ice 2004), sample size (Bernard and Killworth 1993), and data entry programs and personal data assistants (PDAs) (Gravlee et al. 2006; Koster 2006). None of these works have focused comparatively, and in detail, on the strengths and limitations of different methods for collecting information on human behavior in the field. Moreover, over the last 10 15 years, there has been considerable refinement in the specific methods used to collect behavioral data (e.g., Koster 2006). This detailed information may be available to researchers familiar with a subset of behavioral research methods, but there has been almost no comparative discussion of the methodological pros and cons across behavioral study approaches.

In this article, we provide very specific methodological guidance for field researchers considering the collection of behavioral data. The impetus for the writing of this article is threefold: First, we recently have been teaching a course on behavioral research methods. This teaching is part of the Short Courses on Research Methods in Cultural Anthropology (SCRM), a program supported by the National Science Foundation (NSF; http:// www.qualquant. net/training/scrm.htm). Teaching this course has convinced us that there is strong interest among a diverse group of teachers, researchers, and practitioners in learning the specifics of how to undertake behavioral research for a wide range of problems and situations. 
Second, we both have undertaken behavioral research on numerous occasions in different field and sociocultural settings. Hames has completed behaviorally oriented field research among the Ye'kwana and Yanomamö (Hames 1987, 1996), and Paolisso has completed similar work among the Yukpa of Venezuela (Paolisso and Sackett 1988), Embu of Kenya (Paolisso et al. 1989; Baksh et al. 1994), and peasant groups in Nepal (Paolisso et al. 2002) and Honduras (Paolisso et al. 1999). In all of these research undertakings, we both grappled with a host of design and implementation challenges.

Finally, it has become clear to us, based on our own behavioral research, that often solutions to one or two key methodological challenges can make the difference between successful or unsuccessful implementation of a behavioral research project.

Based on our teaching and field research experiences, we argue that a fundamental methodological question researchers must first confront is whether to collect reports of behavior or observations of behavior. As most existing methodological reviews focus on either one or the other, there is limited comparative information available that would help researchers developing behavioral research projects evaluate the strengths and limitations of these two major approaches. In this article, we compare the methodological strengths and weaknesses of collecting reports of behavior versus collecting actual observations of behavior. More specifically, we compare two very frequently used methods to collect reports and observations: time diary research (reports) and instantaneous sampling (observation). Our goal is to provide researchers with information that will help them decide which of the approaches is better suited to their research needs.

In the past, a comparative review of time diary and instantaneous sampling would perhaps not have been necessary. Time dairies were used mainly by sociologists and demographers working in literate, Western populations where respondents could be contacted through mail or telephone and could either verbally report or complete the time diary themselves. The result was large, representative national samples (Stinson 1999).

In contrast, instantaneous sampling was used more by anthropologists working alone in small communities in non-Western cultural settings. Here, there were no national-level census data from which to develop a sampling frame, but there were local communities or groups that could be censused and households that could be visited randomly.

Today, however, these historical differences have been greatly reduced. In technologically advanced countries, researchers from different disciplines are using cell phones, pagers, and handheld computers to "beep" respondents at randomly selected times to collect immediate recall data on be- 
havior as well as respondent's emotional and psychological states (Weisner et al. 2001; Gravlee et al. 2006). In non-Western settings, anthropologists have trained field staff to implement instantaneous sampling (Paolisso et al. $1989,1999,2002)$. Many of the communities anthropologists once studied are now closely linked to mainstream societies and have higher levels of literacy and thus are more amenable to time diary methods.

To help us compare the time diary and instantaneous sampling, we begin with a description of the history, use, and methodological steps involved in each approach. We next move beyond background description to a discussion of some key methodological similarities and differences between the two approaches. We conclude with a comparative overview of the most significant strengths and limitations of time diary and instantaneous sampling.

\section{Behavioral Recall and Direct Observations of Behavior}

Behavioral researchers are interested in identifying the range and diversity of activities undertaken, the temporal dimensions of the activities (e.g., time allocated to each activity and when and for how long the activity occurred), and context (e.g., the location of activity and if the activity was undertaken alone or in conjunctions with other activities) (Robinson and Godbey 1999). We begin with a description of origins, methodological steps, and types of results produced by time diary and instantaneous sampling.

\section{Behavioral Recall Using Time Diaries}

Perhaps, the most widely used and known approach for collecting systematic information on human behavior is recall. In behavioral recall, individuals are asked to report their activities for a specified period of time in the recent past. Methods to collect behavioral recall data can be grouped into two large categories. The first consists of open-ended questions, included for example in a survey, that ask respondents to estimate the time they spent in specific activities. The respondent needs to remember: (1) if they engaged in activity " $x$ " during the study period and (2) if so, for how long (Robinson and Godbey 1999). Although easy to administer, this recall approach has been criticized because often respondents: (1) do not remember all their behaviors; (2) overestimate the time spent in activities, resulting in more minutes allocated to activities than actually available in the reference period; (3) use varying understanding of activities (watching 
TV while cooking gets reported as TV by one respondent and cooking by another; and (4) underreport socially undesirable or unacceptable behavior (Robinson and Godbey 1999).

The second approach to collecting recall of behavior is the use of time diaries. "The time diary is a micro-behavioral technique for collecting self-reports of an individual's behavior in an open-ended fashion on an activity-by-activity basis" (Robinson and Godbey 1999:66). In a time diary, "a verbatim description of the day's activities is collected along with an assignment of the approximate starting and stopping times for each activity, recorded either in free format or in fixed 5- to 10-minute intervals" (Stinson 1999:14).

Data from time diary studies have been used to advance a wide range of social science research, including trends and gender differentials in housework (Bianchi et al. 2000), parental time with children (Sandberg and Hofferth 2001; Sayer et al. 2004), and overall leisure (Schor 1991; Robinson and Godbey 1999; Jacobs and Gerson 2004). Other time diary studies have investigated trends in TV viewing, Internet use, and specific types of leisure activities (Robinson and Godbey 1999), civic involvement (Putman 2000; Sayer 2001), and religious participation (Presser and Stinson 1998).

Time diaries are an established approach within the social sciences and one for which there has been extensive methodological discussions (Robinson and Godbey 1999). In the United States, the use of time diaries can be traced to work by the United States Department of Agriculture (USDA) in the 1920s and 1930s to create daily time records for homemakers (Stinson 1999). In the early 20th century, anthropologist Audrey Richards collected time use diaries among the Bemba of Zimbabwe (then Rhodesia) (Richards 1939). There is an equally long history of time diary studies in Europe, Canada, and Australia (Stinson 1999). An extensive and well-known time diary study is the "Multinational Time Budget Study," undertaken by Szalai (1972). The time diary method has been used widely in surveys of behaviors and time allocation among Americans (Robinson 1976; Robinson and Godbey 1999). An exemplary and ongoing time diary study is the U.S. government's Bureau of Labor Statistics American Time Use Study (ATUS; www.bls.gov/ tus/; Horrigan and Herz 2004).

In time diaries, the most commonly used recall period is the previous 24 hours. More distant recall periods are also possible, such as last week or month, although they are used much less often than the 24-hour recall. The general rule is that the more distant the recall period, the more general will be the recall. 
Once the recall period is determined, the next methodological step is to use a chronological framework to assist respondents in remembering their activities for that period. In the USDA studies, a 12-hour clock was used. Participants were instructed to draw lines on the clock diagram to mark the beginning and ending times of their activities and to describe the activity inside the intervening spaces (see Exhibit 1 in Stinson 1999).

Today, most studies use a chronological listing of activities rather than a clock. There are two formats for these listing. First, beginning at a specified time (e.g., 4:00 a.m. or "the time you woke up"), the respondent is asked to list the activities she or he engaged in for specified increments of time during the day, for example, every 15 minutes. Alternatively, past activities can be elicited using a less-structured chronological listing. Rather than prompting behavioral recall for small intervals of time, respondents are asked to list the activities they undertook in the order they completed them, starting from a specified time (e.g., midnight or 4:00 a.m.). In this approach, the beginning and ending times are dictated by the respondents' reported length of time they spent in each behavior. There is less respondent burden in this approach, although the interviewer has less control over the recall process.

The asking of behavior for specified periods of time produces fine-grained data, if interviewers and respondents can manage the cognitive burden of recalling behavior in such small segments. Piloting is critical to determine the optimal time interval, which should be the smallest possible that guides respondents through the day's activities without creating mental fatigue and loss of recall accuracy. The target individual provides the verbatim report of his or her activity, which is then coded.

Most of the time, diary studies use coding schemes based on the structure developed by Szalai (1972). These activity codes are typically arranged into mutually exclusive behavior groups that cover all aspects of human activity. These generally include personal care, employment, education, domestic work, child care, purchasing goods and services, voluntary work, social and community activities, recreation and leisure, and travel. For example, the ATUS coding lexicon uses a hierarchical structure, classifying reported activities into 17 major categories, with two additional levels of detail in each category. ATUS coders assign a six-digit classification code to each diary activity (rather than the three-digit code commonly used in other time-use surveys). The first two digits represent the major activity categories; the next two digits represent the second-tier level of detail; the final two digits represent the third-the most detailed level of activity (Shelley 2005). The website http://www.bls.gov/tus/lexicons.htm contains the ATUS codes and instructions. 
The ATUS collects information on time spent in each of more than 400 detailed activities. A data extract builder (ATUS-X) has been designed to make it easy for users to create data files that contain the time use, personal characteristic, and household characteristic variables they want, thus making the data more accessible to a broader audience. The output from the ATUS-X is a data file consisting of person-level records that contain the variables a user has requested and formatted for direct input into SAS, Stata, or SPSS statistical programs.

\section{Behavioral Observation Using Instantaneous Sampling}

There is a robust literature on behavioral observation methods in psychology, animal behavior, and anthropology. General reviews can be found in Altmann's classic article (1974), the textbook of Martin and Bateson (1993), and, in anthropology, works by Gross (1984), Johnson and Sackett (1998), Borgerhoff Mulder and Caro (1985), and Hames (1992). By direct observation, we mean observations collected by a researcher in contrast to time diary or other recall studies in which the subject reports or records his or her behavior. Any behavioral observation is a combination of who and what is observed and whether the behavior is recorded continuously or instantaneously.

Behavioral observations have been fundamental to economic and ecological research in anthropology that tests hypotheses about the energetics of human adaptation and develops measures for an adequate characterization of production systems and resource use patterns (Hames 1992). Behavioral observations have also, for example, figured importantly in evaluating the determinants of food exchange in egalitarian society (Gurven 2004); the role of skill versus strength in the achievement of adult productivity (Bock and Sellen 2002); productivity throughout the life course and intergenerational wealth flow (Kaplan 1994); tests of optimal foraging models (Hawkes et al. 1982); and the allocation of alloparental care (Kramer 2005).

Although ethnographers use the full range of behavioral observation techniques as described by Martin and Bateson (1993), we will focus only on instantaneous sampling because of its dominance in anthropological research (for a more comprehensive review, see Hames In press). Instantaneous sampling, as the name suggests, simply records the behavior of the individual the instant he or she is observed. Commonly called "spot checks" (after Johnson 1975), "scan sampling" or "instantaneous scan sampling" (Borgerhoff Mulder and Caro 1985; Hames 1992), instantaneous sampling is by far the most commonly used method in ethnography. The procedure con- 
sists of recording a subject's behavior the moment the subject is observed. In community-based ethnographic studies, it usually consists of serially visiting households in a community or section of a community and recording the behavior of everyone present at the moment (instant) that the individual is viewed by the researcher.

Although the goal of this method is to record behavior observed by the researcher, this requirement is met in various ways. If the person to be recorded is not present during the sampling period, the solution is for the researcher to ask someone present where the absent subject is and what he or she is doing. Consequently, the "observation" is really a report making the data point equivalent to the recall or report approach described above. When this occurs, the researcher needs to note that the observation is really a report and must, as practicable, ensure that the report is accurate (Borgerhoff Mulder and Caro 1985; Hames 1992).

An important goal of instantaneous sampling is to generate a random sample of naturally occurring behaviors. To accomplish this, researchers randomize the time of the start of their observations, where they start, and the route they take through a settlement. These choices are made in advance of the observational round and are typically generated by a table of random numbers before the observations are made. These directives are followed, regardless of weather conditions or the likelihood of interesting events that may occur in the future. Adherence to a rigid set of protocols is necessary to avoid biasing observations toward behaviors that are easily visible or behaviors that the researcher believes are interesting, important, extraordinary, or rare. Borgerhoff Mulder and Caro (1985), Hawkes et al. (1987), and Hames (1992) describe sources of bias when observational protocols are not followed.

Instantaneous sampling is a "dimensionless" measure as it has no duration. The only statistics that can be compiled are counts of the various behaviors recorded but such counts can be legitimately transformed into realtime measures. For example, if one samples behavior during waking hours, say a 14 -hour day, and one knows that $15 \%$ of observations were in food preparation activities, then one could reasonably conclude that 2.1 hours per day were spent in this activity (e.g., see Hames 1992 and Gurven and Kaplan 2006).

Instantaneous recording has a number of advantages. First, compared to focal follows or continuous monitoring, it is very economical in terms of an ethnographer's research time. An outcome of this economy is that it permits a large number of different individuals to be sampled. In some cases, over the course of a year, ethnographers working alone have averaged more 
than 300 observations per person in a village of more than 100 (Hames 1987; Flinn 1988; Paolisso et al. 1989). Finally, it is less obtrusive to subjects, so they are less likely to modify their behavior compared to the constant scrutiny of continuous observation.

Researchers using instantaneous sampling have used both functional and structural descriptors in codes (Borgerhoff Mulder and Caro 1985; Hames 1992). Structural descriptions of behavior describe the bodily actions, stances, orientations, and so on of the observed and can be quite detailed because one may be describing a very complex pattern of behavior in a sentence-like form. Functional descriptions focus on the purpose or design of the behavior, are simple, and conform to our intuitive understanding of behavior. An excellent discussion of these two types of coding schemes can be found in Borgerhoff Mulder and Caro (1985:327-28) and should be read by anyone planning to observe behavior. Finally, Johnson and Sackett (1998) present a set of standardized, functional cross-cultural behavioral codes used by a number of researchers engaged in observational research.

\section{Methodological Comparison: Time Diary and Instantaneous Sampling}

In this section, we compare and contrast time diary and instantaneous sampling in terms of (1) the type of samples they produce and how those samples capture variability in individual behavior; (2) how each method collects information on the activities of other individuals interacting with the target individual; and (3) how each method handles the recording of the frequent situation when the target individual is engaged in multiple activities at once.

\section{Sample Differences: Average Day versus Average Individual}

Time diary and instantaneous sampling can both produce large samples of behavior. However, sample differences between the two methods result from implicit assumptions researchers have about the sources of variability in individual behavior. In time diary, there is a sampling bias toward routine, daily behaviors; in instantaneous sampling, there is a sampling bias toward individual behaviors over a longer period of time than a 24-hour day.

The sample unit for time diary is typically the 24-hour day. Individuals are asked to recall activities for the previous 24 hours and provide verbatim recalls of their activities, along with some time estimates. Although the 
individuals report specific activities, these individual activities form part of a larger sequence of continuous and linked activities. To varying degrees, preceding and proceeding activities influence the likelihood of an activity occurring, the time of day when it occurs, and its duration. For example, if an individual is ill and in bed, then many of the rest of the day's activities will be constrained by these early behaviors or individual conditions. Also of importance is the case that most time diary studies collect only one 24-hour recall period per individual. The resulting sample is cross-sectional in nature, comparing ( $n$ ) number of individuals' 24-hour day of activities. There is no repeat of any individual's behavior for another time period. Finally, the number of activities recalled per individual for his or her 24-hour day depends on how active or inactive they were on the selected study day. If, for example, an individual is sick and in bed with the flu, then there will most likely be fewer activities reported with greater amounts of time spent in each activity, on average. The 24 -hour period needs to be fully accounted for.

Given the above sampling strategy, time diaries produce valid and reliable descriptions of daily, repeated, and routine behaviors. The behaviors that individuals must do or often do with great regularity are reported more frequently and with greater accuracy than rare behaviors. The result is a good assessment of the typical day of activities for the study individual. However, time diary studies do not capture so well the infrequent behaviors, the spikes of activities that fall outside the normal, daily routine. For example, parental activities with dependent children are fairly well captured in time diaries because parental time investment occurs on a daily basis. However, time diaries do not capture as well infrequent or irregular life cycle transitions, for example, birth, graduation, church confirmation, marriage, job change, and retirement.

In addition, time diaries may not be able to capture important household, family, or community transfers, such as time devoted to the care of older parents. Large portions of the care provided to elders can occur in short periods of time and be crisis-driven and thus hard to capture adequately in the 24-hour time diary or even in a 2-day diary format. Such research may require that parent-child pairs are sampled and the diaries recorded when the mother is laid up.

In contrast, instantaneous sampling produces estimates not for the average day but for the average individual. Typically, in instantaneous sampling, the sampling period is much longer than 1 or 2 days and often covers an entire year, to account for seasonal variation in behavior. Instead of disaggregating a stream of continuous and often linked behavior into indi- 
vidual activities (codes), instantaneous sampling involves completing multiple snapshots of individual behaviors over the study period. Because significant time periods can elapse between "snapshots," often days, there is no temporal link between each observed activity. Each observation is a behavior for the target individual on different days or months. The resulting individual sample is longitudinal: Individual behavior can be compared at different points. Aggregating the individual observations produces data on the typical individual's behavior over time, although often the data are analyzed as cross-sectional. Finally, all individuals have the same number of observations, which is determined by how often the behavior snapshots are taken.

The sampling strategy in instantaneous sampling is useful for capturing the variability in behavior that occurs over longer periods than an average day. Still, because the observations are made during the day (see below), the behavior recorded is used to estimate an individual's average daily behavior. This is true even though the observation is dimensionless, with no reported or measured time spent by any individual in any activity. Instantaneous sampling does produce descriptions of the average individual's daily activity pattern, including those activities that are infrequent and rare and not routine but important. The result is a description of the relative importance of different behaviors to an individual expressed in a comparison of the amount of time he or she spent in each activity. It produces less of what the average day is like, but more what is the relative importance of different activities to an individual, measured by the amount of time that individual spends in those activities, on average. (Remember the base is not an average day.) Compared to the time diary, its strength is that it does produce descriptions of daytime behavior and time allocation in capturing the infrequent and atypical behaviors: These behaviors will show up as a small percentage of the total observations. The result is a good proxy for the average day.

A weakness of the sampling strategy of instantaneous sampling arises from one difficulty in implementing the approach. If people move around, then instantaneous sampling may be inefficient because they are absent when the researchers visit to observe. However, as noted above, many researchers work around this problem by asking those present to report on the whereabouts and activities of the missing person, thus allowing the researcher to complete the visit/observation of all members of the group (e.g., household).

The use of reports instead of direct observation creates problems of its own. The person reporting what an absent person is doing may classify the behavior differently than the researcher and/or the report may be what 
the informant thought the absent individual intended to do. There are solutions to this problem such as contacting the absent person to ask what they were doing while absent. Regardless, it is important that the researcher notes whether the observation was a true observation or a report (Borgerhoff Mulder and Caro 1985).

Finally, instantaneous sampling is typically made during daytime hours and sometimes is extended into early evening or morning. In many places, sampling during nighttime hours is either dangerous or unwelcome. The importance of sampling during nighttime hours likely varies from culture to culture (Scaglion 1986). In industrial settings, it is likely to be required to gain an unbiased set of observations. If nighttime is important, then the researcher is advised to do some time diary for the evening period.

To summarize, with time diary research, the sample is for the typical day, a 24-hour period. With instantaneous sampling, the sample is for the typical individual. The choice of which sampling frame better captures behavior variability is an ethnographic decision: how patterned is behavior into either routine days versus seasonal activities and how important are nonroutine, infrequent life events to the research. Is the day the base unit from which to compare individual behavior or is there a need for a more open and longer time frame from which to sample?

\section{Location and Behavior of Others}

Often, the meaning or significance of specific behavioral changes depends on the spatial, demographic, or sociocultural contexts. Both time diary and instantaneous observation methodologies include the collection of some forms of location, demographic, or sociocultural context information such as age, sex, family membership, occupation, education, and so on. How time diary and instantaneous methods allow the collection of context information is an important consideration for researchers planning to use one or the other method.

Information on the location of the activity can be useful for a variety of questions, such as garden labor exchange (Hames 1987) and interhousehold meal sharing (Hames and McCabe 2007). In both cases, when someone was observed to work in a garden not his or her own or eat a meal in another household, the owner of the field or household was known, and these cases were recorded as labor and food exchanges, respectively.

Researchers recognize that individuals do not act alone much of the time. They also understand that many important behaviors are the product of joint time allocation among two or more individuals. Yet, the focus 
of the time diary approach is collection and analysis of data for one individual, for one representative 24-hour period. Time diary approaches may collect information on other individuals present, but the primary reason to collect that additional information is to code accurately the behavior of the target individual. Generally, insufficient information is collected to code and analyze the behavior of the other individuals, at a level comparable to the target individual.

The reluctance of time diary researchers to collect information on other individuals is explained in part by the difficulties in data collection and analysis. For example, most interviewing approaches (e.g., phone interview) preclude the possibility of collecting time diaries from an entire household, as trying to make contact with all household members on the same day is nearly impossible (Stinson 1999). In addition, some statisticians argue that collecting clusters of interrelated, individual behaviors increases survey standard errors because of the endogenous effects of these interindividual activities. Others researchers argue the opposite that the social dynamism produced by the intertwining of household members' activities demands that households be studied as a group (Stinson 1999). There is further concern that if data are collected for more than one member of a household or social group, response rates could suffer overall, due to the difficulty of scheduling and completing the time diary interview (Schwartz et al. 2002).

Three data collection options are available to time diary researchers interested in collecting information on more than one individual. First, for each recalled activity, the interviewer can ask if anyone else was present or involved. The data recording form used by the Australian Time Use Survey has a column for recording the informant's response to the question "Who was with you at home, or with you away from home (e.g., no-one, family, friends?)" (Stinson 1999).

A second approach is to develop specific submodules of data collection, focused, for example, on specific behaviors that are defined by a strong interactive component, such as child care. In these instances, a separate interviewing guideline is developed that seeks detailed information on who was present, did what, and for how long. Although limited to a subdomain of behavior, this approach allows the inclusion of more and diverse individuals, undertaking a wide range of behaviors.

Finally, a third approach is to use open-ended questions. In the 1992 and 1998 Canadian General Social Survey, diary information was collected from one respondent per household, using a retrospective telephone interview. The Canadian survey augments the data from the diary by also asking re- 
spondents direct, stylized questions about their own and their partners' use of time in several unpaid activities (Winkler 2002).

Instantaneous sampling has also been used to chart human interaction, but most of the research has been limited to the study of infant-caretaker interactions, a frequent, observable and important activity crucial in studies of developmental psychology, socialization, and parental investment (see Hewlett 1992 and Hewlett and Lamb 2005). Nevertheless, studies of interactions should be extended beyond parent-child interactions. Despite its potential utility for elucidating patterns of social organization, very few researchers use it to study social interaction behaviorally. Exceptions include Flinn's study (1988) of conflict between fathers and daughters and their prospective suitors, under the heading of daughter guarding; Sugawara's (1988) study of intercamp visiting among the San; Kimura's (1992) analysis of association patterns among the Bongando of Zaire; and the study by Johnson and Johnson (1976) of husband and wife interactions among the Machiguenga.

In instantaneous sampling, there are several different ways to collect information on interaction. In studies of child care, the child is the focal subject and those providing care are added to the record, followed by the kind of care or behavior they are directing toward the child. In the end, the caregiver has his or her behavior coded as care-giving, followed by the name of the subject receiving care followed by the kind of care being received (Hewlett 1989). The same basic procedure may be followed for any other kind of interaction. If the number of people interacting in a group is large, data entry can become quite tedious. A solution to this problem is to classify the behavior as an interaction, and the interactants will be defined having the same date, time, and location when their entries are recorded. Finally, interactions are often asymmetric (talking-listening or feeding-being fed), and codes may be modified to note such asymmetries.

\section{Recording Simultaneous Activities}

A third major methodological challenge for time diary and instantaneous sampling researchers is whether and how to collect information on more than one activity for the target individual. Time diary research suggests that individuals may spend 3-4 hours per day engaged in multiple activities (Stinson 1999). As Szalai has remarked, "any time-budget study which does not grapple in some way with the problem of recording secondary or parallel activities is essentially unable to give a balanced account of the great variety of activities which fill up everyday life" from (Stinson 1999:18). 
The importance of capturing more than one activity of the target individual is particularly pronounced in the area of child care. Both time diary and instantaneous sampling methods have included information on the multiple activities of care providers.

Typically, time diary studies allow respondents to report at least one "simultaneous" or "secondary" activity to their perceived main activity.

For instantaneous sampling, one can easily add secondary and tertiary activities to the record. For example, in observing the Ye'kwana, it was not uncommon for Hames to come upon a woman sitting on the lever of a manioc press while nursing a child and conversing with an adjacent woman. Does the researcher code for food preparation, child care, social interaction, or for all three activities? Johnson and Sackett (1998:327) call this coding challenge the simultaneity problem and describe the strengths and weaknesses of six possible solutions. All their solutions are reasonable, but the one we favor is to preserve the richness of the observation and code the behaviors as primary, secondary, and tertiary.

\section{Conclusions}

In this article, we reviewed two well-known and used methods for the systematic study of human behavior: time diary and instantaneous sampling. Our goal has been to describe each approach in sufficient detail so that future behavioral researchers would be able to understand the basics of each approach, along with some of their methodological strengths and weaknesses. We also compared both approaches in terms of their sampling biases, and how each method handles the challenges of recording multiple behaviors of and context information for the individual under study. We also focused on how time diary and instantaneous observation capture the behaviors of other individuals, as it affects the behavior of the target respondent.

The strengths of instantaneous observations include accuracy and high-resolution behavioral descriptions. Because the observer is recording behavior, the observation is more likely to be accurate and it can have as much detail as needed to meet the researcher's study goals. The context of the behavior can be as fully characterized as desired and simultaneous behaviors can be recorded in full detail. Finally, given that individuals are repeatedly sampled, one can easily produce profiles of how time is allocated over a long period of time and how it varies with social characteristics. 
A particular weakness of instantaneous behavioral observations is the inability to create measures of duration of behaviors or behavioral sequences. (These two weaknesses can be overcome using continuous observations of behavior.) The method can be time consuming, as the researcher and not the subject must record the behavior. In addition, it is intrusive and can be challenging to implement in the field, although most researchers who have used the approach have developed successful approaches well suited to their field research conditions. Finally, the presence of an observer has the potential to alter the behavior of the subject and subject's absence may force the research to rely on reports instead of observations.

The time diary method has a number of methodological strengths. First, there is a well-established methodological literature available as well as ongoing discussion of the methodological issues of time diary research. With a moderate amount of effort, a novice behavioral researcher can access and even participate in current methodological discussions, all of which should be of great guidance in developing and adapting time diary methods to any particular survey or ethnographic situation. There are also extensive coding schemes and databases of coded behavior available that, with little or no modification, can be used in new research (see ATUS).

A second strength of the time diary approach is that, if it is feasible to use, it is a very efficient method for collecting information on daily, routine behaviors, including their sequence and duration. If feasibility is a critical consideration here, participants must be willing to work with the investigator to produce a time diary that is detailed, accurate, and covers the entire specified period of study. This contrasts sharply with the feasibility requirements of instantaneous observations, where, after initial data collection conditions are satisfied, the researcher controls many of the implementation steps. However, if feasible, the time diary method is a very efficient method at collecting reliable and comparable data on behaviors, including most important information on duration and sequence. This efficiency enables the method to produce large, representative samples.

In terms of weaknesses, the time diary method is less well suited to collect information on multiple activities and context information, particularly, in the latter case, information on the behaviors of others who are interacting with the study individual. For anthropologists and other social scientists interested in group dynamics and more holistic, descriptive accounts of behavior, this limitation can be significant. Time diary researchers have developed additional data collection modules that add insights on key areas of behavioral interaction, but these are often partial and cover only a limited range of behaviors and interactions (e.g., child care). In addition, as men- 
tioned above, time diary has sampling biases that results in better information on routine, daily behaviors, and less information on the infrequent or irregular behavior. To us, this bias is a key consideration in deciding whether to use time diary versus instantaneous observation.

To conclude, ultimately the approach that one selects will depend on the questions asked and the nature of the population sampled. Behavioral observations have been indispensable in traditional ethnographic settings where literacy is absent and/or subjects are unaccustomed to handling survey forms. It is also indispensable for subjects who are young and where the specifics of the behavior being studied can only be accurately identified by trained experts. Time diaries have worked best in literate populations and when the collection of a large sample of daily activities is desired. Importantly, it produces data on behavioral sequences and duration that are difficult to obtain using direct observation. Unlike direct observations, the accuracy of time diaries depends on the training, reliability, and motivation of subjects to make accurate, candid, and timely reports of their behaviors.

In the future, the use of PDAs and other electronic recording devices holds considerable promise because of their ability to combine the strengths of observations and reports. Like behavioral observations, recording of behavior is done in real time (whenever the device requests input), thus enhancing accuracy by avoiding recall error; it is relatively unobtrusive; and it can collect instantaneous or continuous observations. Finally, it collects data in a digital form, allowing rapid statistical analysis to track trends and potential errors in research protocols. For future behavioral researchers, the methodological issue will increasingly become not whether to use time diary or instantaneous observation, for example, but how to combine the bests of both approaches to improve validity, reliability, and relevance of behavioral measurements to increasingly complex and diverse social science questions.

Acknowledgments - The authors thank Dr. Russ Bernard for his valuable guidance and feedback in developing our behavioral research methods course, which is part of the Short Courses on Research Methods in Cultural Anthropology (SCRM), and the course's students for their many insightful comments that have helped them continue to improve it. The authors received no financial support for the research and authorship of this article and declared no conflicts of interest with respect to its publication. 


\section{References}

Altmann, J. 1974. The observational study of behavior. Behaviour 48:1-41.

Baksh, M. 1989. Spot observations technique in time allocation research [Part 1]. CAM: Cultural Anthropology Methods Newsletter 1:1-3.

Baksh, M. 1990. Spot observations technique of time allocation research [Part 2]. CAM: Cultural Anthropology Methods Newsletter 2:6-7.

Baksh, M., C. Neumann, M. Paolisso, R. Trostle, and A. Jansen. 1994. The influence of reproductive status on rural Kenyan women's time use. Social Science and Medicine 39:345-54.

Bernard, H. R., and P. D. Killworth. 1993. Sampling in time allocation research. Ethnology 32:207-15.

Bianchi, S., M. Milkie, L. Sayer, and J. Robinson. 2000. Is anyone doing the housework? Trends in the gender division of household labor. Social Forces 79:191-228.

Bock, J., and D. Sellen. 2002. Childhood and the evolution of the human life course: Introduction. Human Nature 13:153-60.

Borgerhoff Mulder, M., and T. Caro. 1985. The use of quantitative observation techniques in anthropology. Current Anthropology 26:232-62.

Flinn, M. 1988. Parent-offspring interactions in a Caribbean village: Daughter guarding. In Human reproductive behaviour, eds. L. Betzig, M. Borgerhoff Mulder, and P. Turke, 189-200. Cambridge: Cambridge University Press.

Gravlee, C., S. Zenk, S. Woodos, Z. Rowe, and A. Schulz. 2006. Handheld computers for direct observation of the social and physical environment. Field Methods 18:382-97.

Gross, D. R. 1984. Time allocation: A tool for the study of cultural behavior. Annual Review of Anthropology 13:519-58.

Gurven, M. 2004. To give and to give not: The behavioral ecology of human food transfers. Behavioral and Brain Sciences 27:120-55.

Gurven, M., and H. Kaplan. 2006. Determinants of time allocation across the lifespan: A theoretical model and an application to the Machiguenga and Piro of Peru. Human Nature: An Interdisciplinary Biosocial Perspective 17:1-49.

Hames, R. 1987. Relatedness and garden labor exchange among the Ye'kwana. Evolution and Human Behavior 8:354-92.

Hames, R. 1989. Time, efficiency, and fitness in the Amazonian protein quest. Research in Economic Anthropology 11:43-85.

Hames, R. 1992. Time allocation. In Evolutionary ecology and human behavior, eds. E. A. Smith and B. Winterhalder, 203-36. Chicago: Aldine de Gruyter. 
Hames, R. 1996. Costs and benefits of monogamy and polygyny for Yanomamo women. Ethology and Sociobiology 17:181-99.

Hames, R. In press. Production decisions and time allocation. In Society and environment: Methods and research design, eds. V. Ismael, E. A. Smith, and S. Aswani. Cambridge: Cambridge University Press.

Hames, R., and C. McCabe. 2007. Meal sharing among the Ye'kwana. Human Nature 18:1-21.

Hawkes, K., K. Hill, H. Kaplan, and A. M. Hurtado. 1987. Some problems with instantaneous scan sampling. Journal of Anthropological Research 43:239-47.

Hawkes, K., K. Hill, and J. O'Connell. 1982. Why hunters gather: Optimal foraging and the Ache of eastern Paraguay. American Ethnologist 9:379-98.

Hewlett, B. S. 1989. Multiple caretaking among African Pygmies. American Anthropologist. 91:186-88.

Hewlett, B. S. 1992. Father-child relations: Cultural and biosocial contexts. New York: Aldine de Gruyter.

Hewlett, B. S., and M. E. Lamb. 2005. Hunter-gatherer childhoods: Evolutionary, developmental, \& cultural perspectives. New Brunswick: Aldine de Gruyter.

Horrigan, M., and D. Herz. 2004. Planning, designing, and executing the BLS American time-use survey. Monthly Labor Review October:3-19.

Ice, G. 2004. Technological advances in observational data collection: The advantages and limitations of computer-assisted data collection. Fields Methods 16:352-75.

Jacobs, J., and K. Gerson. 2004. The time divide. Cambridge, MA: Harvard University Press.

Johnson, A. 1975. Time allocation in a Machiguenga community. Ethnology 14:301-10.

Johnson, A., and O. Johnson. 1976. Male/female relations and the organization of work in a Machiguenga community. American Ethnologist 2:634-48.

Johnson, A., and R. Sackett. 1998. Direct systematic observation of behavior. In Handbook of methods in cultural anthropology, eds. H. R. Bernard, 301-30. Walnut Creek, CA: AltaMira.

Kaplan, H. 1994. Evolutionary and wealth flows theories of fertility: Empirical tests and new models. Population and Development Review 20:753-91.

Kimura, D. 1992. Daily activities and social association of the Bongando in central Zaire. African Study Monographs 13:1-33.

Koster, J. 2006. The use of the Noldus Observer 5.0 and Psion handheld computer in a remote fieldwork setting. Field Methods 18:430-36. 
Kramer, K. L. 2005. Children's help and the pace of reproduction: Cooperative breeding in humans. Evolutionary Anthropology 14:224-37.

Martin, P., and P. Bateson. 1993. Measuring behavior. Cambridge: Cambridge University Press.

Minge-Klevana, W. 1980. Does labor time decrease with industrialization? A survey of time-allocation studies. Current Anthropology 21:279-98.

Paolisso, M., M. Baksh, and J. Thomas. 1989. Women's agricultural labor, child care and infant diarrhea in rural Kenya. In Women's work and Child Welfare in the Third World, eds. J. Leslie and M. Paolisso, 217-36. Boulder, CO: Westview.

Paolisso, M., K. Hallman, L. Haddad, and S. Regmi. 2002. Does cash crop adoption detract from child care provision? Evidence from rural Nepal. Economic Development and Culture Change 50:313-33.

Paolisso, M., and R. Sackett. 1988. Traditional meat procurement strategies among the Irapa-Yukpa of the Venezuela-Colombia border area. Research in Economic Anthropology 7:177-202.

Paolisso, M., S. Gammage, and L. Casey. 1999. Gender and household-level responses to soil degradation in Honduras. Human Organization 58:261-73.

Presser, S., and L. Stinson. 1998. Data collection mode and social desirability bias in self-reported religious attendance. American Sociological Review 63:137-45.

Putman, R. 2000. Bowling alone. New York: Simon and Schuster.

Richards, A. 1939. Land, labour, and diet in Northern Rhodesia. London: Oxford University Press.

Robinson, J. P. 1976. Changes in American's use of time, 1965-1975. Cleveland: Communication Research Center.

Robinson, J. P., and G. Godbey. 1999. Time for life: The surprising ways Americans use their time. University Park: The Pennsylvania State University Press.

Sandberg, J., and S. Hofferth. 2001. Changes in children's time with parents: United States, 1981-1997. Demography 38:423-36.

Sayer, L. 2001. Time use, gender and inequality: Differences in men's and women's market, nonmarket and leisure time. Unpublished dissertation, College Park, University of Maryland.

Sayer, L., S. Bianchi, and J. Robinson. 2004. Are parents investing less in children? Trends in mothers' and fathers' time with children. American Journal of Sociology 107:1-43.

Scaglion, R. 1986. Importance of nighttime observations in time allocation studies. American Ethnologist 13:537-45.

Schor, J. 1991. The overworked American: The unexpected decline of leisure. New York: Basic Books. 
Schwartz, L., D. Herz, and H. Frazis. 2002. Measuring intrahousehold allocation of time: Response to Anne E. Winkler. Monthly Labor Review February:53-9.

Shelley, K. 2005. Developing the American time use survey activity classification system. Monthly Labor Review June:3-15.

Stinson, L. 1999. Measuring how people spend their time: A time-use survey design. Monthly Labor Review August:12-19.

Sugawara, K. 1988. Visiting relations and social interactions between residential groups of the central Kalahari San: Hunter-gatherer camp as a micro-territory. African Study Monographs 8:173-211.

Szalai, A. 1972. The use of time: Daily activities of urban and suburban populations in twelve countries. The Hague: Mouton.

Weisner, T., G. W. Ryan, L. Reese, K. Kroesen, L. Bernheimer, and R. Gallimore. 2001. Behavior sampling and ethnography: Complementary methods for understanding home-school connections among Latino immigrant families. Field Methods 13:20-46.

Winkler, A. 2002. Measuring time use in multiple-person households. Monthly Labor Review February:45-52. 